\title{
NEW TAXA IN SECTION PETOTA OF SOLANUM
}

\author{
C.M. Ochoa
}

Genetic Resources Department, International Potato Center (CIP), P.O. Box 1558, Lima, PERU

\section{ABSTRACT}

Seven new taxa are described in Solanum sect. Petota.

KEY WORDS: Solanaceae, Solanum, new species

This paper is the result of a review that I have been doing of many herbarium specimens during the preparation of a monograph on Peruvian tuberbearing Solanum. Although the new proposed taxa are of an intraspecific level, I considered it necessary to publish them prior to the publication of the monograph. These taxa will be fully illustrated in my forthcoming book.

\section{Solanum series Acaulia}

Solanum acaule Bitt. f. incuyo Ochoa, forma nova. TYPE: PERU. Dept. Ayacucho: prov. Parinacochas, Crusvuelta, $3400-3500 \mathrm{~m}$ alt., ca. Incuyo, Aprilis 1975, C. Ochoa \& A. Salas 9834 (HOLOTYPUS: OCH; Isotypi: $\mathrm{CIP}, \mathrm{OCH})$.

Planta rosulata. Folia simplicia et pinnatifida lobis late decurrentibus secum rachim, terminali latiori ovato-lanceolato vel late elliptico-lanceolato. Corolla pallide lilacina.

\section{Solanum series Conicibaccata}

Solanum chromatophilum Bitt. var. subnivale Ochoa, var. nov. TYPE: PERU. Dept. Ancash: prov. Yungay, supra Huishca, $4300 \mathrm{~m}$ alt., circa conterminus nivis perpetuus, Aprilis 17, 1978, C. Ochoa 12084 (HOLOTYPUS: $\mathrm{OCH})$. 
Planta a species typica differs: folia modice secta tantum 1-2 paribus lateralibus foliorum, sine interfoliolis, pedicello circa media parte vel infra articulato; corollae majore praebentes, stellatae vel substellatae, lobis vix vel non nisi acuminatis.

Solanum chromatophilum Bitt. f. sausianense Ochoa forma nov. TYPE: PERU. Dept. Huanuco: prov. Huamalies, circa Sausian, $3580 \mathrm{~m}$ alt., Aprilis 26, 1967, C. Ochoa 2646 (HOLOTYPUS: OCH).

A species typica primordialiter differs: forma et magnitudine foliolorum plerumque majoris et angustior lanceolatis ad apicem acutis vel acuminatis, et basi petiolulatis; itidem planta robustior, statura superans magis profuse ramosa, folia minus incisa.

\section{Solanum series Megistacroloba}

Solanum megistacrolobum Bitt.f. purpureum Ochoa, forma nov. TYPE: PERU. Dept. Arequipa: prov. Cailloma, Montis Yahuarmayo, $4000 \mathrm{~m}$ alt., ca. Pulpera, Martio 6, 1981, C. Ochoa 14273(HOLOTYPUS: OCH; Isotypi: CIP,MOL,USM).

Forma a typo, tuberculorum forma colore magnitudineque admodum variabilis differt. Tuberculi plerumque lati, complanati vel cylindracei cum peridermis purpureis nitidisque. Baccae copiosissime albo punctata.

\section{Solanum series Piurana}

Solanum chiquidenum Ochoa var. gracile Ochoa, var. nov. TYPE: PERU. Dept. Cajamarca: prov. Cutervo, Valle Grande, $2500 \mathrm{~m}$ alt. inter Santa Rosa de Tingo et Laguna Negra, Junius 1983, C. Ochoa \& Alberto Salas 15229 (HOLOTYPUS: OCH).

Planta gracilis, cauli tenui parce ramoso. Folia 2-3 paribus loborum sin inter rachim interlobulis. Lobi anguste longeque lanceolati apice acuminato marginibus erosis. Corolla alba, stellata vel substellata. 
Solanum chiquidenum Ochoa var. robustum Ochoa, var. nov. TYPE: PERU. Dept. Cajamarca: prov. Contumaza, Montis Cunanten, $2900 \mathrm{~m}$ alt., inter Chorrillo et La Ramada, Maius 1982, C. Ochoa 14760 (HOLOTYPUS: OCH; Isotypi: CIP,MOL).

Planta robusta, caule crasso valde ramoso. Folia quam typus species maiora et magis dissecta, 2-3 paribus foliorum plus 2-4 paribus interloborum; lobis elliptico lanceolatis, apice acuto breviter acuminato marginibus loborum erosis ciliatisque. Corolla albo-eburnea, rotacea.

Solanum chiquidenum Ochoa $\mathrm{f}$. amazonense Ochoa, forma nov. TYPE: PERU. Dept. Amazonas: prov. Luya, vicinitas Cuelap, $2800 \mathrm{~m}$ alt., Maius 15, 1967, C. Ochoa 2679 (HOLOTYPUS: OCH).

Planta quam species typica Solanum chiquidenum Ochoa multo brevior. Folia breviora, plerumque pari foliorum latioribus subsessilibus et lobo terminali latiori late elliptico-lanceolato et cum corolla albo-eburnea, stellata vel substellata a typo differt. 


\section{$2 \mathrm{BHL}$ Biodiversity Heritage Library}

Ochoa, C M. 1994. "New taxa in section Petota of Solanum." Phytologia 77, 390-392. https://doi.org/10.5962/bhl.part.4443.

View This Item Online: https://www.biodiversitylibrary.org/item/47116

DOI: https://doi.org/10.5962/bhl.part.4443

Permalink: $\underline{\text { https://www.biodiversitylibrary.org/partpdf/4443 }}$

\section{Holding Institution}

New York Botanical Garden, LuEsther T. Mertz Library

\section{Sponsored by}

The LuEsther T Mertz Library, the New York Botanical Garden

\section{Copyright \& Reuse}

Copyright Status: In copyright. Digitized with the permission of the rights holder.

Rights Holder: Phytologia

License: http://creativecommons.org/licenses/by-nc-sa/3.0/

Rights: https://biodiversitylibrary.org/permissions

This document was created from content at the Biodiversity Heritage Library, the world's largest open access digital library for biodiversity literature and archives. Visit BHL at https://www.biodiversitylibrary.org. 